\title{
A New Probability Model Based on a Coherent System with Applications
}

\author{
Christophe Chesneau $\mathbb{D}^{1},{ }^{1}$ Hassan S. Bakouch $\mathbb{D}^{2},{ }^{2}$ Bilal A. Para $\mathbb{D},^{3}$ \\ and Mohammad H. Poursaeed (iD ${ }^{4}$ \\ ${ }^{1}$ Department of Mathematics, LMNO, Campus II, Science 3, Université de Caen, 14032 Caen, France \\ ${ }^{2}$ Department of Mathematics, Faculty of Science, Tanta University, Tanta, Egypt \\ ${ }^{3}$ Department of Mathematical Sciences, Islamic University of Science and Technology, Awantipora, JeK, India \\ ${ }^{4}$ Department of Statistics, Lorestan University, Khorramabad, Iran
}

Correspondence should be addressed to Christophe Chesneau; christophe.chesneau@gmail.com

Received 4 January 2022; Accepted 7 January 2022; Published 13 February 2022

Academic Editor: Aya Essam

Copyright (C) 2022 Christophe Chesneau et al. This is an open access article distributed under the Creative Commons Attribution License, which permits unrestricted use, distribution, and reproduction in any medium, provided the original work is properly cited.

\begin{abstract}
The notion of a coherent system allows us to formalize how the random lifetime of the system is connected to the random lifetimes of its components. These connections are also generators of new pliant distributions, being those of various mixes of minimum and maximum of random variables. In this paper, a new four-parameter lifetime probability distribution is introduced by using the notion of a coherent system. Its structural properties are assessed and evaluated, including the analytical study of its main functions, stochastic dominance results, moments, and moment generating function. The proposed distribution, in particular, is proving to be efficient at fitting data with slight negative skewness and platykurtic as well as leptokurtic nature. This is illustrated by the analysis of three relevant real-life data sets, two in reliability and another in production, exhibiting the significance of the introduced model in comparison to various well-known models in statistical literature.
\end{abstract}

\section{Introduction}

Coherent systems are very important in reliability theory and data analysis. A n-component system is said to be coherent if its structure function is monotonic (that is, the improvement of components cannot lead to a deterioration in system performance), and it contains no irrelevant components (that is, all components have an effect on system performance). The detailed descriptions of various coherent systems can be found in [1-3]. Many authors have studied the reliability properties of coherent systems. Special attention has been paid to independent and identically distributed coherent systems and, in particular, to $k$ out-of- $n$ (order statistics), parallel, and series systems. Recently, some authors have started to study systems with dependence structures (see, for example, [4-7]). In this study, the following coher- ent system is considered. Consider a system having three components, numbered by $I, I I$, and $I I I$, the component $I I$ $I$ playing a central role. Assume that the components $I, I I I$ , and $I I$ are ordered in a straight line, and that the system works if there are at least two consecutively working components, i.e., IN $\mapsto(I) \mapsto(I I I) \rightleftharpoons(I I) \mapsto$ OUT, where for instance, $(I) \rightleftharpoons(I I)$ means that $I$ and $I I I$ are consecutive and connected; if one of the two component falls, their connection ends. This system is evoked in [8] as a consecutive 2 -out-of-3 system. Hence, the lifetimes of the components $I$, $I I$, and III can be modeled as three random variables, say $U_{1}, U_{2}$, and $U_{3}$, respectively. Here, we suppose that they are independent and subjected to the following distributional assumptions: $U_{1}$ follows the exponential distribution with parameter $\lambda_{1}>0, U_{2}$ follows the exponential distribution with parameter $\lambda_{2}>0$, and $U_{3}$ follows the Weibull 


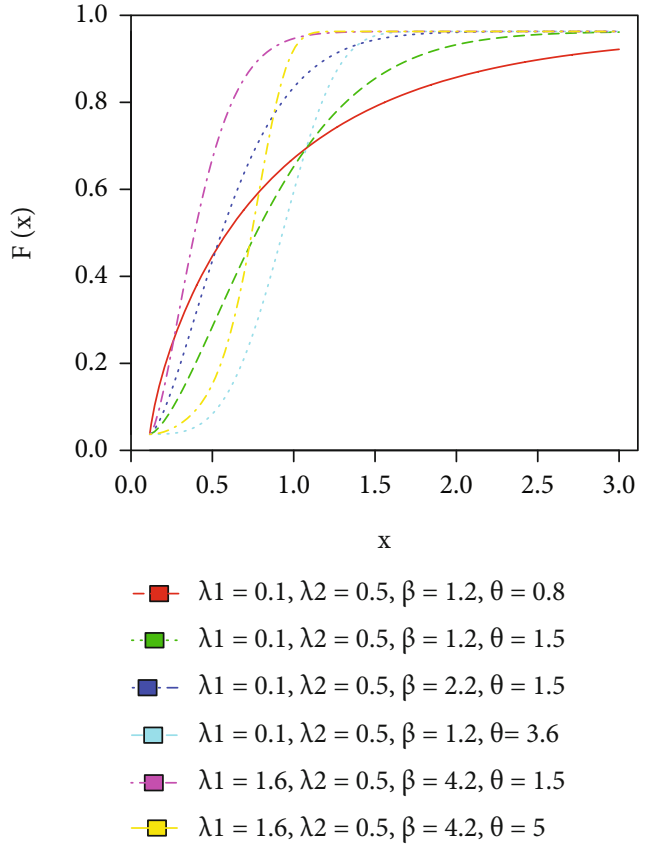

Figure 1: Plots of the cdf for different combinations of the distribution parameters.

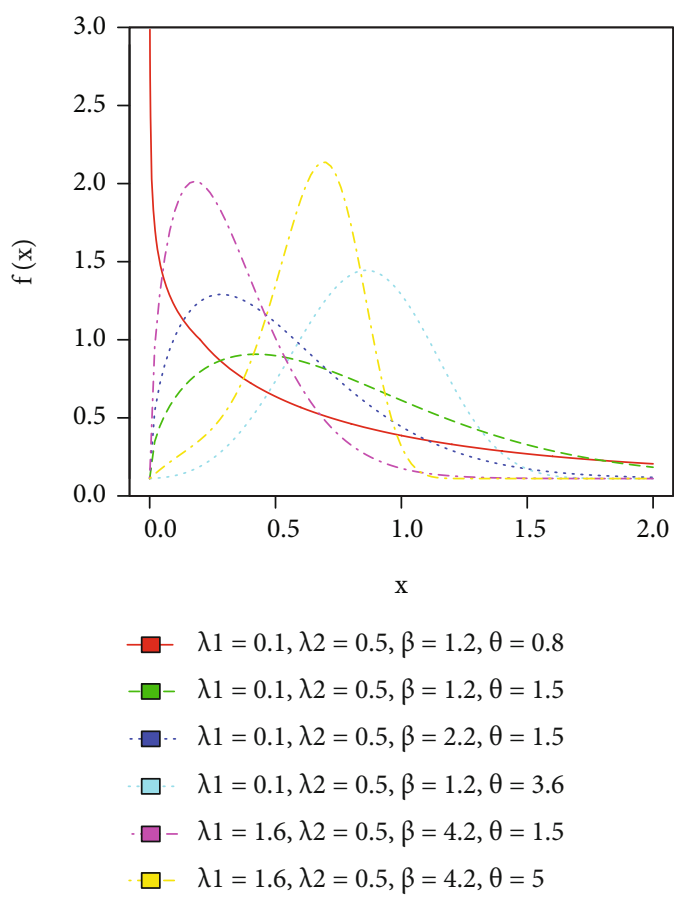

FIgURe 2: Plots of the pdf for different combinations of the distribution parameters.

distribution with scale parameter $\beta>0$ and shape parameter $\theta>0$. That is, their respective cumulative distribution functions (cdfs) are given as

$F_{U_{1}}(x)=1-e^{-\lambda_{1} x}, F_{U_{2}}(x)=1-e^{-\lambda_{2} x}, F_{U_{3}}(x)=1-e^{-\beta x^{\theta}}, x>0$, and all equals 0 otherwise. Note that the distribution of $U_{3}$ is supposed to be more flexible to the other because of the pivotal role of the component $I I I$ in the system; if $I I I$ falls first, the lifetime of the system is $U_{3}$. More generally, the lifetime of the system can be modeled by a random variable $T$ such that

$$
T=\max \left[\min \left(U_{1}, U_{3}\right), \min \left(U_{2}, U_{3}\right)\right] .
$$

Here, the four-parameter distribution of $T$ is called the special coherent system (SCS) distribution. To our knowledge, the SCS distribution is not listed in the literature, despite its simple physical interpretation and the potential for various statistical purposes. The objective of this study is to explore the basics of the SCS distribution, beginning with the study of its determinant functions, such as the cumulative distribution function, probability density function (pdf) and hazard rate function (hrf). In particular, we emphasize the fact that the SCS distribution is adapted to fit data having various skewness and kurtosis natures. Several results on stochastic ordering are discussed. Also, the moments and moment generating functions are developed. Then, the inference for the SCS model is explored through the use of the maximum likelihood method. We show how the SCS model can be applied quite efficiently to fit three important real-life data sets, being more relevant in comparison to various well-known models in the statistical literature.

The paper is divided into the following sections. Section 2 describes the main interesting functions of the SCS distribution along with a graphical analysis. Some of its properties are discussed in Section 3. The parametric inference is studied in Section 4, with simulation studies to verify the performance of the obtained estimates. Section 5 ends the practical study of the SCS distribution by showing how it can be applied to analyze three data sets. Section 6 ends the paper with a conclusion.

\section{Functions}

In this section, the main functions of the SCS distribution are presented. We recall that any random variable following the SCS distribution can be expressed as (2). We thus logically denote such a random variable as $T$. The cdf of $T$ is expressed in the result below.

Proposition 1. The cdf of $T$ can be expressed as

$$
F_{T}(x)=1-e^{-\beta x^{\theta}}\left(e^{-\lambda_{1} x}+e^{-\lambda_{2} x}-e^{-\left(\lambda_{1}+\lambda_{2}\right) x}\right), x>0
$$

and it equals to 0 if $x \leq 0$.

Proof. Let $S_{U_{1}}(x)=e^{-\lambda_{1} x}, S_{U_{2}}(x)=e^{-\lambda_{2} x}, S_{U_{3}}(x)=e^{-\beta x^{\theta}}, x>0$, and all equals to 1 , otherwise, be the survival functions of $U_{1}$ , $U_{2}$, and $U_{3}$, respectively. By using all the assumptions made on $U_{1}, U_{2}$, and $U_{3}$, by applying diverse standard probabilistic results, for $x>0$, we get 


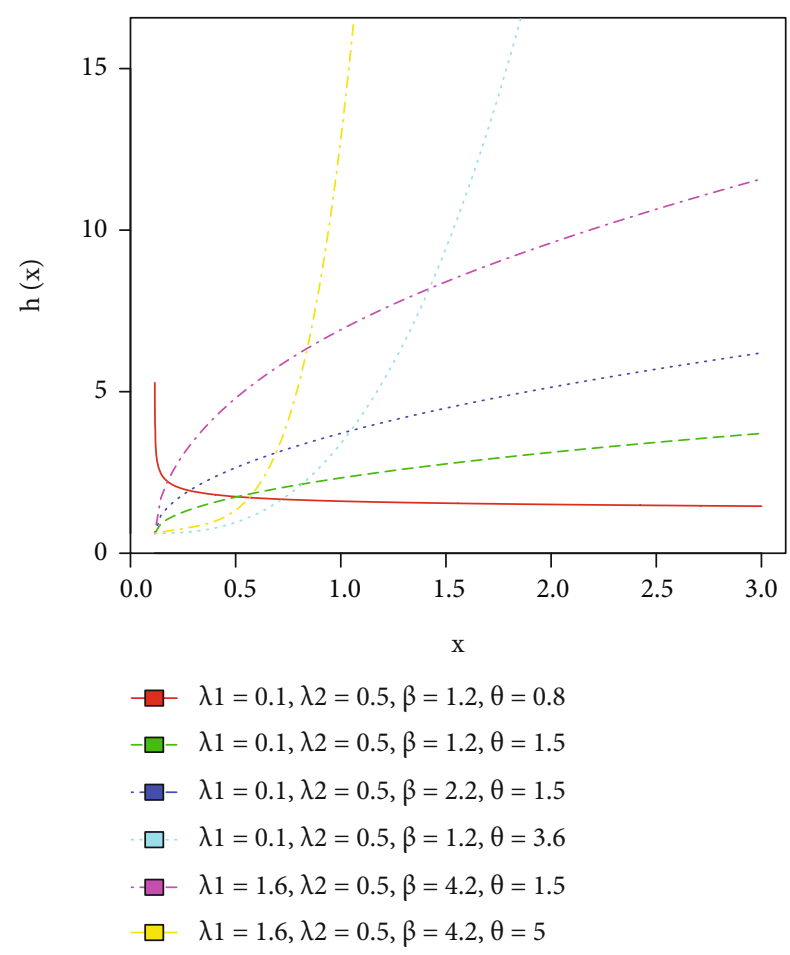

FIgURE 3: Plots of the hrf for different combinations of the distribution parameters.

$$
\begin{aligned}
F_{T}(x) & =P(T \leq x)=P\left\{\max \left[\min \left(U_{1}, U_{3}\right), \min \left(U_{2}, U_{3}\right)\right] \leq x\right\} \\
& =P\left[\min \left(U_{1}, U_{3}\right) \leq x \text { and } \min \left(U_{2}, U_{3}\right) \leq x\right] \\
& =1-P\left[\min \left(U_{1}, U_{3}\right)>x \text { or } \min \left(U_{2}, U_{3}\right)>x\right] \\
& =1-\left[P\left(U_{1}>x, U_{3}>x\right)+P\left(U_{2}>x, U_{3}>x\right)-P\left(U_{1}>x, U_{2}>x, U_{3}>x\right)\right] \\
& =1-S_{U_{3}}(x)\left[S_{U_{1}}(x)+S_{U_{2}}(x)-S_{U_{1}}(x) S_{U_{2}}(x)\right] \\
& =1-e^{-\beta x^{\theta}}\left(e^{-\lambda_{1} x}+e^{-\lambda_{2} x}-e^{-\left(\lambda_{1}+\lambda_{2}\right) x}\right) .
\end{aligned}
$$

This ends the proof of Proposition 1.

Figure 1 gives plots of $F_{T}(x)$ for different combinations of the distribution parameters.

Figure 1 demonstrates that the cdf curve of the SCS distribution is pliant enough to present concave or convex shapes.

As second important function, the pdf of the SCS distribution is obtained as, for $x>0, f_{T}(x)=F_{T}^{\prime}(x)$, that is

$$
\begin{aligned}
f_{T}(x)= & e^{-\beta x^{\theta}}\left[\left(\beta \theta x^{\theta-1}+\lambda_{1}\right) e^{-\lambda_{1} x}+\left(\beta \theta x^{\theta-1}+\lambda_{2}\right) e^{-\lambda_{2} x}\right. \\
& \left.-\left(\beta \theta x^{\theta-1}+\lambda_{1}+\lambda_{2}\right) e^{-\left(\lambda_{1}+\lambda_{2}\right) x}\right]
\end{aligned}
$$

and it equals to 0 otherwise. Thus, one can write $f_{T}(x)$ as $f_{T}(x)=w(x) f_{U_{3}}(x)$, where

$$
\begin{gathered}
w(x)=\left(1+\frac{\lambda_{1}}{\beta \theta} x^{1-\theta}\right) e^{-\lambda_{1} x}+\left(1+\frac{\lambda_{2}}{\beta \theta} x^{1-\theta}\right) e^{-\lambda_{2} x} \\
-\left(1+\frac{\lambda_{1}+\lambda_{2}}{\beta \theta} x^{1-\theta}\right) e^{-\left(\lambda_{1}+\lambda_{2}\right) x}, x>0,
\end{gathered}
$$

and it equals to 0 otherwise. Hence, $f_{T}(x)$ is a weighted version of the Weibull distribution with parameters $\beta$ and $\theta$.

As immediate properties, for $\theta<1$, we have $\lim _{x \rightarrow 0} f_{T}($ $x)=+\infty$; for $\theta=1$, we have $f_{T}(0)=\beta$ and, for $\theta>1$, we have $\lim _{x \longrightarrow 0} f_{T}(x)=0$. In all cases, we have $\lim _{x \longrightarrow+\infty} f_{T}(x)=0$.

Figure 2 presents plots of $f_{T}(x)$ for different combinations of the distribution parameters.

From Figure 2, we see that the pdf curve can be decreasing or has right skewed as well as left skewed bell shapes. Also, various kurtosis properties are observed. These observations make the related model ideal for the fit of various lifetime data.

Based on (3), the survivor function of the SCS distribution is obtained as $S_{T}(x)=1-F_{T}(x)$; that is,

$$
S_{T}(x)=e^{-\beta x^{\theta}}\left(e^{-\lambda_{1} x}+e^{-\lambda_{2} x}-e^{-\left(\lambda_{1}+\lambda_{2}\right) x}\right), x>0,
$$

and it equals to 1 otherwise. Also, based on (5) and (7), the hrf of the SCS distribution can be expressed as $h_{T}(x)=f_{T}($ $x) / S_{T}(x)$, that is, after some developments,

$$
h_{T}(x)=\beta \theta x^{\theta-1}+\frac{\lambda_{1} e^{-\lambda_{1} x}+\lambda_{2} e^{-\lambda_{2} x}-\left(\lambda_{1}+\lambda_{2}\right) e^{-\left(\lambda_{1}+\lambda_{2}\right) x}}{e^{-\lambda_{1} x}+e^{-\lambda_{2} x}-e^{-\left(\lambda_{1}+\lambda_{2}\right) x}}, x>0 \text {, }
$$

and it equals to 0 otherwise. Therefore, we can write $h_{T}(x)$ $=\beta h_{U_{3}}(x)+w_{*}(x)$, where

$$
w_{*}(x)=\frac{\lambda_{1} e^{-\lambda_{1} x}+\lambda_{2} e^{-\lambda_{2} x}-\left(\lambda_{1}+\lambda_{2}\right) e^{-\left(\lambda_{1}+\lambda_{2}\right) x}}{e^{-\lambda_{1} x}+e^{-\lambda_{2} x}-e^{-\left(\lambda_{1}+\lambda_{2}\right) x}}, x>0,
$$

and it equals to 0 otherwise, and $h_{U_{3}}(x)$ denotes the hrf of $U_{3}$.

We can see how the new system can make the hrf of the Weibull distribution more flexible because $w_{*}(x)$ is a nonmonotonic function that depends on $\lambda_{1}$ and $\lambda_{2}$. Figure 3 gives plots of the hrf for different combinations of the distribution parameters.

In Figure 3, we observe that the hrf presents increasing, decreasing, and $J$ and shapes which are of interest for modeling various lifetime data.

\section{Properties}

Here, some properties of the SCS distribution are investigated. We recall that $T$ denotes a random variable following the SCS distribution, i.e., as defined in (2), and with the cdf specified by (3).

3.1. Stochastic Dominance. Some stochastic dominance properties of the SCS distribution are described in the proposition below. 
TABLE 1: Simulation study of MLEs for the proposed model.

\begin{tabular}{|c|c|c|c|c|c|c|c|c|c|}
\hline \multirow{2}{*}{ Parameter } & \multirow{2}{*}{$n$} & \multicolumn{4}{|c|}{$\lambda_{1}=0.1, \lambda_{2}=0.2, \beta=0.1, \theta=0.3$} & \multicolumn{4}{|c|}{$\lambda_{1}=0.5, \lambda_{2}=0.8, \beta=0.6, \theta=0.9$} \\
\hline & & Bias & Var & MSE & $\mathrm{CP}$ & Bias & Var & MSE & $\mathrm{CP}$ \\
\hline$\lambda_{1}$ & \multirow{4}{*}{25} & 0.021114 & 0.002115 & 0.002561 & 0.889 & 0.205723 & 2.193071 & 2.235393 & 0.919 \\
\hline$\lambda_{2}$ & & -0.03953 & 0.062169 & 0.063732 & 0.921 & 0.132916 & 0.690794 & 0.70846 & 0.911 \\
\hline$\beta$ & & 0.042505 & 0.008775 & 0.010581 & 0.941 & 0.000567 & 0.123177 & 0.123178 & 0.951 \\
\hline$\theta$ & & 0.116879 & 0.222573 & 0.236234 & 0.917 & 0.017071 & 0.095244 & 0.095535 & 0.917 \\
\hline$\lambda_{1}$ & \multirow{4}{*}{50} & -0.008200 & 0.001106 & 0.001174 & 0.939 & 0.32076 & 0.617408 & 0.720295 & 0.929 \\
\hline$\lambda_{2}$ & & 0.036686 & 0.05765 & 0.058996 & 0.942 & 0.044022 & 0.232803 & 0.234741 & 0.922 \\
\hline$\beta$ & & 0.015211 & 0.00755 & 0.007781 & 0.946 & -0.1001 & 0.075348 & 0.085368 & 0.933 \\
\hline$\theta$ & & 0.122715 & 0.199639 & 0.214698 & 0.925 & -0.12145 & 0.060964 & 0.075715 & 0.937 \\
\hline$\lambda_{1}$ & \multirow{4}{*}{100} & -0.00087 & 0.001028 & 0.001028 & 0.949 & -0.00044 & 0.15383 & 0.153830 & 0.942 \\
\hline$\lambda_{2}$ & & 0.010637 & 0.01313 & 0.013243 & 0.952 & 0.12464 & 0.206504 & 0.222039 & 0.949 \\
\hline$\beta$ & & -0.01046 & 0.001511 & 0.00162 & 0.948 & -0.01268 & 0.075396 & 0.075557 & 0.967 \\
\hline$\theta$ & & 0.173295 & 0.114213 & 0.144244 & 0.955 & -0.03226 & 0.054359 & 0.0554 & 0.951 \\
\hline$\lambda_{1}$ & \multirow{4}{*}{300} & 0.013048 & 0.00065 & 0.00082 & 0.959 & 0.082777 & 0.04763 & 0.054482 & 0.956 \\
\hline$\lambda_{2}$ & & 0.010905 & 0.009139 & 0.009258 & 0.957 & 0.094535 & 0.114553 & 0.12349 & 0.955 \\
\hline$\beta$ & & -0.00537 & 0.001055 & 0.001084 & 0.949 & -0.06717 & 0.030705 & 0.035217 & 0.951 \\
\hline$\theta$ & & 0.151536 & 0.108245 & 0.131208 & 0.965 & -0.04193 & 0.013846 & 0.015605 & 0.959 \\
\hline$\lambda_{1}$ & \multirow{4}{*}{500} & 0.002621 & 0.000482 & 0.000489 & 0.967 & 0.008584 & 0.049028 & 0.049102 & 0.959 \\
\hline$\lambda_{2}$ & & 0.006849 & 0.001234 & 0.00128 & 0.977 & -0.04306 & 0.063105 & 0.064959 & 0.966 \\
\hline$\beta$ & & -0.00772 & 0.001023 & 0.001083 & 0.973 & 0.03503 & 0.020659 & 0.021886 & 0.972 \\
\hline$\theta$ & & 0.043243 & 0.083642 & 0.085512 & 0.962 & 0.004247 & 0.008681 & 0.008699 & 0.959 \\
\hline
\end{tabular}

Proposition 2. Let $F_{T}\left(x ; \beta, \lambda_{1}, \lambda_{2}, \theta\right)=F_{T}(x)$ be (3). Then, the following stochastic dominances hold:

(i) For $\beta^{*} \geq \beta$, we have $F_{T}\left(x ; \beta, \lambda_{1}, \lambda_{2}, \theta\right) \leq F_{T}\left(x ; \beta^{*}\right.$, $\left.\lambda_{1}, \lambda_{2}, \theta\right)$

(ii) For $\lambda_{1}^{*} \geq \lambda_{1}$, we have $F_{T}\left(x ; \beta, \lambda_{1}, \lambda_{2}, \theta\right) \leq F_{T}(x ; \beta$, $\left.\lambda_{1}^{*}, \lambda_{2}, \theta\right)$

(iii) For $\lambda_{2}^{*} \geq \lambda_{2}$, we have $F_{T}\left(x ; \beta, \lambda_{1}, \lambda_{2}, \theta\right) \leq F_{T}(x ; \beta$, $\left.\lambda_{1}, \lambda_{2}^{*}, \theta\right)$

(iv) For $\theta^{*} \geq \theta$ and $x \geq 1$, we have $F_{T}\left(x ; \beta, \lambda_{1}, \lambda_{2}, \theta\right) \leq$ $F_{T}\left(x ; \beta, \lambda_{1}, \lambda_{2}, \theta^{*}\right)$, and for $x<1$, the reversed inequality holds

Proof. Firstly, these inequalities are straightforward for $x \leq 0$. The first three inequalities are equivalent to say that $F_{T}(x$; $\left.\beta, \lambda_{1}, \lambda_{2}\right)$ is an increasing function with respect to $\beta, \lambda_{1}$, and $\lambda_{2}$, independently. For $x>0$, the following results hold:

$$
\frac{\partial}{\partial \beta} F_{T}\left(x ; \beta, \lambda_{1}, \lambda_{2}\right)=x^{\theta}\left(e^{\lambda_{1} x}+e^{\lambda_{2} x}-1\right) e^{-\left(\lambda_{1}+\lambda_{2}\right) x} e^{-\beta x^{\theta}}>0,
$$

implying that $F_{T}\left(x ; \beta, \lambda_{1}, \lambda_{2}\right)$ is an increasing function with respect to $\beta$,

$$
\frac{\partial}{\partial \lambda_{1}} F_{T}\left(x ; \beta, \lambda_{1}, \lambda_{2}\right)=x\left(e^{\lambda_{2} x}-1\right) e^{-\left(\lambda_{1}+\lambda_{2}\right) x} e^{-\beta x^{\theta}}>0,
$$

implying that $F_{T}\left(x ; \beta, \lambda_{1}, \lambda_{2}\right)$ is an increasing function with respect to $\lambda_{1}$, and

$\frac{\partial}{\partial \lambda_{2}} F_{T}\left(x ; \beta, \lambda_{1}, \lambda_{2}\right)=x\left(e^{\lambda_{1} x}-1\right) e^{-\left(\lambda_{1}+\lambda_{2}\right) x} e^{-\beta x^{\theta}}>0$,

implying that $F_{T}\left(x ; \beta, \lambda_{1}, \lambda_{2}\right)$ is an increasing function with respect to $\lambda_{2}$.

For the last inequality, note that

$\frac{\partial}{\partial \theta} F_{T}\left(x ; \beta, \lambda_{1}, \lambda_{2}\right)=\beta x^{\theta} \log (x)\left(e^{\lambda_{1} x}+e^{\lambda_{2} x}-1\right) e^{-\left(\lambda_{1}+\lambda_{2}\right) x} e^{-\beta x^{\theta}}$.

All the main multiplicative terms are positive, except $\log (x)$. Therefore, the sign of this derivative function is the one of $\log (x)$. Hence, $F_{T}\left(x ; \beta, \lambda_{1}, \lambda_{2}\right)$ is an increasing function with respect to $\theta$ for $x \geq 1$ and a decreasing function with respect to $\theta$ for $x<1$. Proposition 2 is proved.

Another stochastic order result involving simple distributions is presented below. 
TABLE 2: Simulation study of MLEs for the proposed model.

\begin{tabular}{|c|c|c|c|c|c|c|c|c|c|}
\hline \multirow{2}{*}{ Parameter } & \multirow{2}{*}{$n$} & \multicolumn{4}{|c|}{$\lambda_{1}=1.5, \lambda_{2}=1.2, \beta=0.9, \theta=0.6$} & \multicolumn{4}{|c|}{$\lambda_{1}=1.6, \lambda_{2}=1.8, \beta=1.4, \theta=1.5$} \\
\hline & & Bias & Var & MSE & $\mathrm{CP}$ & Bias & Var & MSE & $\mathrm{CP}$ \\
\hline$\lambda_{1}$ & \multirow{4}{*}{25} & 0.258307 & 1.685182 & 1.751905 & 0.929 & -0.67831 & 1.260407 & 1.720518 & 0.828 \\
\hline$\lambda_{2}$ & & -0.105120 & 0.534711 & 0.545761 & 0.879 & -0.0423 & 2.246578 & 2.248367 & 0.914 \\
\hline$\beta$ & & 0.077258 & 0.262241 & 0.268210 & 0.944 & 0.568665 & 0.643097 & 0.966477 & 0.953 \\
\hline$\theta$ & & -0.008806 & 0.051804 & 0.051882 & 0.937 & 0.451302 & 1.185069 & 1.388742 & 0.913 \\
\hline$\lambda_{1}$ & \multirow{4}{*}{50} & 0.244534 & 1.407408 & 1.467205 & 0.938 & -0.50457 & 0.917448 & 1.172044 & 0.927 \\
\hline$\lambda_{2}$ & & -0.101305 & 0.222697 & 0.232960 & 0.951 & 0.044837 & 1.742389 & 1.744399 & 0.942 \\
\hline$\beta$ & & 0.056540 & 0.110326 & 0.113523 & 0.947 & 0.394625 & 0.52356 & 0.679289 & 0.939 \\
\hline$\theta$ & & 0.048513 & 0.018310 & 0.020664 & 0.948 & 0.207914 & 0.215991 & 0.259219 & 0.932 \\
\hline$\lambda_{1}$ & \multirow{4}{*}{100} & 0.223024 & 1.346181 & 1.395921 & 0.956 & -0.6562 & 0.544443 & 0.975047 & 0.949 \\
\hline$\lambda_{2}$ & & 0.026269 & 0.196364 & 0.197054 & 0.953 & -0.04021 & 1.638775 & 1.640392 & 0.942 \\
\hline$\beta$ & & -0.059397 & 0.100119 & 0.103647 & 0.958 & 0.385053 & 0.402029 & 0.550295 & 0.968 \\
\hline$\theta$ & & -0.012319 & 0.015320 & 0.015472 & 0.956 & 0.061853 & 0.037724 & 0.04155 & 0.953 \\
\hline$\lambda_{1}$ & \multirow{4}{*}{300} & 0.226485 & 0.818427 & 0.869723 & 0.961 & -0.17858 & 0.232734 & 0.264625 & 0.959 \\
\hline$\lambda_{2}$ & & 0.063117 & 0.082361 & 0.086345 & 0.958 & -0.46086 & 1.023646 & 1.236038 & 0.959 \\
\hline$\beta$ & & -0.002488 & 0.073408 & 0.073414 & 0.951 & 0.475944 & 0.226687 & 0.453209 & 0.962 \\
\hline$\theta$ & & 0.000355 & 0.012194 & 0.012194 & 0.963 & -0.01319 & 0.00942 & 0.009594 & 0.965 \\
\hline$\lambda_{1}$ & \multirow{4}{*}{500} & 0.186137 & 0.582542 & 0.617189 & 0.975 & -0.13091 & 0.106946 & 0.124083 & 0.963 \\
\hline$\lambda_{2}$ & & -0.088530 & 0.021287 & 0.029126 & 0.977 & 0.083023 & 0.818039 & 0.824932 & 0.961 \\
\hline$\beta$ & & -0.064670 & 0.015944 & 0.020126 & 0.973 & 0.091008 & 0.13488 & 0.143163 & 0.971 \\
\hline$\theta$ & & -0.005711 & 0.001958 & 0.001991 & 0.971 & 0.033865 & 0.005222 & 0.006369 & 0.958 \\
\hline
\end{tabular}

TABle 3: Descriptive statistics of data set 1.

\begin{tabular}{lccccccc}
\hline Mean & Variance & SD & Minimum & Maximum & Skewness & Kurtosis & Index of dispersion \\
\hline 1.447 & 0.256 & 0.506 & 0.031 & 2.585 & -0.164 & 3.236 & 0.177 \\
\hline
\end{tabular}

TABle 4: Descriptive statistics of data set 2.

\begin{tabular}{lccccccc}
\hline Mean & Variance & SD & Minimum & Maximum & Skewness & Kurtosis & Index of dispersion \\
\hline 0.469 & 0.037 & 0.192 & 0.017 & 0.878 & -0.335 & 2.686 & 0.079 \\
\hline
\end{tabular}

TABle 5: Descriptive statistics of data set 3.

\begin{tabular}{ccccccccc}
\hline Mean & Variance & SD & Minimum & Maximum & Skewness & Kurtosis & Index of dispersion \\
\hline 1.507 & 0.1051 & 0.324 & 0.550 & 2.240 & -0.899 & 3.923 & 0.069 \\
\hline
\end{tabular}


TABLE 6: MLEs along with standard errors of the parameters in parentheses for the fitted models for data set 1.

\begin{tabular}{|c|c|c|c|c|c|}
\hline Model & & & MLEs & & \\
\hline \multirow{2}{*}{$\operatorname{SCS}\left(\lambda_{1}, \lambda_{2}, \beta, \alpha\right)$} & $\widehat{\lambda}_{1}=0.05$ & $\widehat{\lambda}_{2}=64.07$ & $\widehat{\beta}=0.15$ & $\widehat{\alpha}=3.63$ & \\
\hline & $(0.041)$ & $(3812)$ & $(0.048)$ & $(0.413)$ & \\
\hline Weibull $(\beta, \lambda)$ & $\widehat{\beta}=3.03(0.029)$ & $\widehat{\lambda}=1.60(0.066)$ & & & \\
\hline Gamma $(s, r)$ & $\widehat{s}=0.291(0.020)$ & $\widehat{r}=4.97(0.820)$ & & & \\
\hline $\operatorname{EED}(\alpha, \lambda)$ & $\widehat{\alpha}=4.99(0.981)$ & $\widehat{\lambda}=1.52(0.156)$ & & & \\
\hline $\operatorname{MWD}(\alpha, \beta, \lambda)$ & $\widehat{\alpha}=0.066(0.021)$ & $\widehat{\beta}=1.182(0.702)$ & $\widehat{\lambda}=1.267(0.478)$ & & \\
\hline GLindley $(\lambda, \alpha)$ & $\widehat{\lambda}=1.898(0.168)$ & $\widehat{\alpha}=4.414(0.889)$ & & & \\
\hline $\operatorname{MBLE}(\lambda, \beta, a, b, c)$ & $\widehat{\lambda}=1.521(2.971)$ & $\widehat{\beta}=2.241(2.562)$ & $\widehat{a}=0.576(0.381)$ & $\widehat{b}=0.548(0.571)$ & $\widehat{c}=0.017(0.041)$ \\
\hline $\operatorname{TLED}(\beta, \theta, \lambda)$ & $\widehat{\beta}=0.041(0.076)$ & $\widehat{\theta}=1.166(0.141)$ & $\widehat{\lambda}=-0.891(0.103)$ & & \\
\hline
\end{tabular}

TABLE 7: ML estimates along with standard errors of the parameters in parentheses for the fitted models for data set 2.

\begin{tabular}{|c|c|c|c|c|c|}
\hline Model & & & MLEs & & \\
\hline $\operatorname{SCS}\left(\lambda_{1}, \lambda_{2}, \beta, \alpha\right)$ & $\widehat{\lambda}_{1}=25.9$ & $\widehat{\lambda}_{2}=0.502$ & $\widehat{\beta}=8.21$ & $\widehat{\alpha}=4.06$ & \\
\hline & $(20.6)$ & $(0.165)$ & $(1.675)$ & $(0.471)$ & \\
\hline Weibull $(\beta, \lambda)$ & $\widehat{\beta}=2.60(0.209)$ & $\widehat{\lambda}=0.52(0.021)$ & & & \\
\hline Gamma $(s, r)$ & $\widehat{s}=0.127(0.017)$ & $\widehat{r}=3.68(0.483)$ & & & \\
\hline $\operatorname{EED}(\alpha, \lambda)$ & $\widehat{\alpha}=3.71(0.565)$ & $\widehat{\lambda}=4.20(0.372)$ & & & \\
\hline $\operatorname{MWD}(\alpha, \beta, \lambda)$ & $\widehat{\alpha}=0.199(0.145)$ & $\widehat{\beta}=0.883(0.377)$ & $\widehat{\lambda}=3.89(0.876)$ & & \\
\hline GLindley $(\lambda, \alpha)$ & $\widehat{\lambda}=4.81(0.382)$ & $\widehat{\alpha}=3.58(0.549)$ & & & \\
\hline $\operatorname{MBLE}(\lambda, \beta, a, b, c)$ & $\widehat{\lambda}=3.39(3.87)$ & $\widehat{\beta}=3.99(5.48)$ & $\widehat{a}=1.037(0.405)$ & $\widehat{b}=4.92(11.12)$ & $\widehat{c}=0.021(0.033)$ \\
\hline $\operatorname{TLED}(\beta, \theta, \lambda)$ & $\widehat{\beta}=0.192(0.299)$ & $\widehat{\theta}=9.66(1.175)$ & $\widehat{\lambda}=-0.688(0.154)$ & & \\
\hline
\end{tabular}

TABLE 8: ML estimates along with standard errors of the parameters in parentheses for the fitted models for data set 3.

\begin{tabular}{|c|c|c|c|c|c|}
\hline \multirow{3}{*}{$\begin{array}{l}\text { Model } \\
\operatorname{SCS}\left(\lambda_{1}, \lambda_{2}, \beta, \alpha\right)\end{array}$} & \multicolumn{5}{|c|}{ MLEs } \\
\hline & $\widehat{\lambda}_{1}=0.265$ & $\widehat{\lambda}_{2}=0.270$ & $\widehat{\beta}=0.025$ & $\widehat{\alpha}=7.042$ & \\
\hline & $(0.835)$ & $(0.854)$ & $(0.016)$ & $(0.988)$ & \\
\hline Weibull $(\beta, \lambda)$ & $\widehat{\beta}=5.780(0.576)$ & $\widehat{\lambda}=1.628(0.037)$ & & & \\
\hline Gamma $(s, r)$ & $\widehat{s}=0.086(0.015)$ & $\widehat{r}=17.39(3.041)$ & & & \\
\hline $\operatorname{EED}(\alpha, \lambda)$ & $\widehat{\alpha}=31.34(9.521)$ & $\widehat{\lambda}=2.611(0.238)$ & & & \\
\hline $\operatorname{MWD}(\alpha, \beta, \lambda)$ & $\widehat{\alpha}=0.012(0.011)$ & $\widehat{\beta}=2.884(1.780)$ & $\widehat{\lambda}=1.831(1.035)$ & & \\
\hline GLindley $(\lambda, \alpha)$ & $\widehat{\lambda}=2.990(0.245)$ & $\widehat{\alpha}=26.17(7.985)$ & & & \\
\hline $\operatorname{MBLE}(\lambda, \beta, a, b, c)$ & $\widehat{\lambda}=1.544(1.276)$ & $\widehat{\beta}=1.876(1.592)$ & $\widehat{a}=1.225(0.494)$ & $\widehat{b}=2.072(1.342)$ & $\widehat{c}=0.005(0.002)$ \\
\hline $\operatorname{TLED}(\beta, \theta, \lambda)$ & $\widehat{\beta}=0.001(0.083)$ & $\widehat{\theta}=1.230(0.129)$ & $\widehat{\lambda}=-1.00(0.032)$ & & \\
\hline
\end{tabular}

Proposition 3. The following inequalities hold:

$$
F_{U_{3}}(x) \leq F_{T}(x) \leq G(x)
$$

where $G(x)$ denotes the cdf of $\min \left(U_{*}, U_{3}\right)$, where $U_{*}$ is a random variable following the exponential distribution with parameter $\lambda_{*}=\min \left(\lambda_{1}, \lambda_{2}\right)$ independent of $U_{3}$.

Proof. The two inequalities are clear for $x \leq 0$. For the left inequality, by the definition of $T$ in (2), we have $\min ($ 
TABLE 9: AD, CVM, and $p$ values for fitted models to data set 1.

\begin{tabular}{lcccccccc}
\hline Criterion & SCS & Weibull & Gamma & EED & MWD & GLindley & MBLE & TLED \\
\hline$K-S$ & 0.0474 & 0.0661 & 0.1265 & 0.1443 & 0.0706 & 0.1347 & 0.0582 & 0.1001 \\
$p$ value $(K-S)$ & 0.9977 & 0.9240 & 0.2193 & 0.1130 & 0.8813 & 0.1637 & 0.9734 & 0.4940 \\
AD statistic & 0.1845 & 0.4522 & 1.9352 & 3.0047 & 0.4625 & 2.6199 & 0.3297 & 1.0221 \\
$p$ value (AD) & 0.9941 & 0.7953 & 0.0999 & 0.0274 & 0.7847 & 0.0431 & 0.9140 & 0.3456 \\
CVM statistic & 0.0208 & 0.0567 & 0.3090 & 0.4875 & 0.0607 & 0.4167 & 0.0512 & 0.1708 \\
$p$ value $(\mathrm{CVM})$ & 0.9964 & 0.8363 & 0.1274 & 0.0425 & 0.8111 & 0.0650 & 0.8702 & 0.3326 \\
\hline
\end{tabular}

TABLE 10: AD, CVM, and $p$ values for fitted models to data set 2 .

\begin{tabular}{lcccccccc}
\hline Criterion & SCS & Weibull & Gamma & EED & MWD & GLindley & MBLE & TLED \\
\hline$K-S$ & 0.0458 & 0.0832 & 0.1364 & 0.1477 & 0.0474 & 0.1441 & 0.0479 & 0.0939 \\
$p$ value $(K-S)$ & 0.9783 & 0.4487 & 0.0374 & 0.0188 & 0.9699 & 0.0236 & 0.9668 & 0.3015 \\
AD statistic & 0.1525 & 1.4840 & 3.8036 & 4.6866 & 0.2199 & 4.4901 & 0.2097 & 1.5311 \\
$p$ value (AD) & 0.9984 & 0.1804 & 0.0109 & 0.0041 & 0.9840 & 0.0051 & 0.9875 & 0.1693 \\
CVM statistic & 0.0228 & 0.1894 & 0.6398 & 0.8125 & 0.0302 & 0.7729 & 0.0298 & 0.2513 \\
$p$ value $(\mathrm{CVM})$ & 0.9938 & 0.2892 & 0.0177 & 0.0067 & 0.9761 & 0.0084 & 0.9773 & 0.1867 \\
\hline
\end{tabular}

TABle 11: AD, CVM, and $p$ values for fitted models to data set 3.

\begin{tabular}{lccccccrr}
\hline Criterion & SCS & Weibull & Gamma & EED & MWD & GLindley & MBLE & TLED \\
\hline$K-S$ & 0.1202 & 0.1522 & 0.2163 & 0.2290 & 0.1410 & 0.2264 & 0.2632 & 0.2625 \\
$p$ value $(K-S)$ & 0.3229 & 0.1078 & 0.0055 & 0.0027 & 0.1632 & 0.0031 & 0.0003 & 0.0003 \\
AD statistic & 0.7213 & 1.2407 & 3.0878 & 4.3368 & 0.9954 & 4.2049 & 5.1046 & 6.3461 \\
$p$ value (AD) & 0.5407 & 0.2524 & 0.0248 & 0.0060 & 0.3594 & 0.0070 & 0.0026 & 0.0007 \\
CVM statistic & 0.1282 & 0.2151 & 0.5662 & 0.7983 & 0.1817 & 0.7720 & 1.0468 & 1.2558 \\
$p$ value $(\mathrm{CVM})$ & 0.4644 & 0.2403 & 0.0268 & 0.0072 & 0.3065 & 0.0083 & 0.0018 & 0.0006 \\
\hline
\end{tabular}

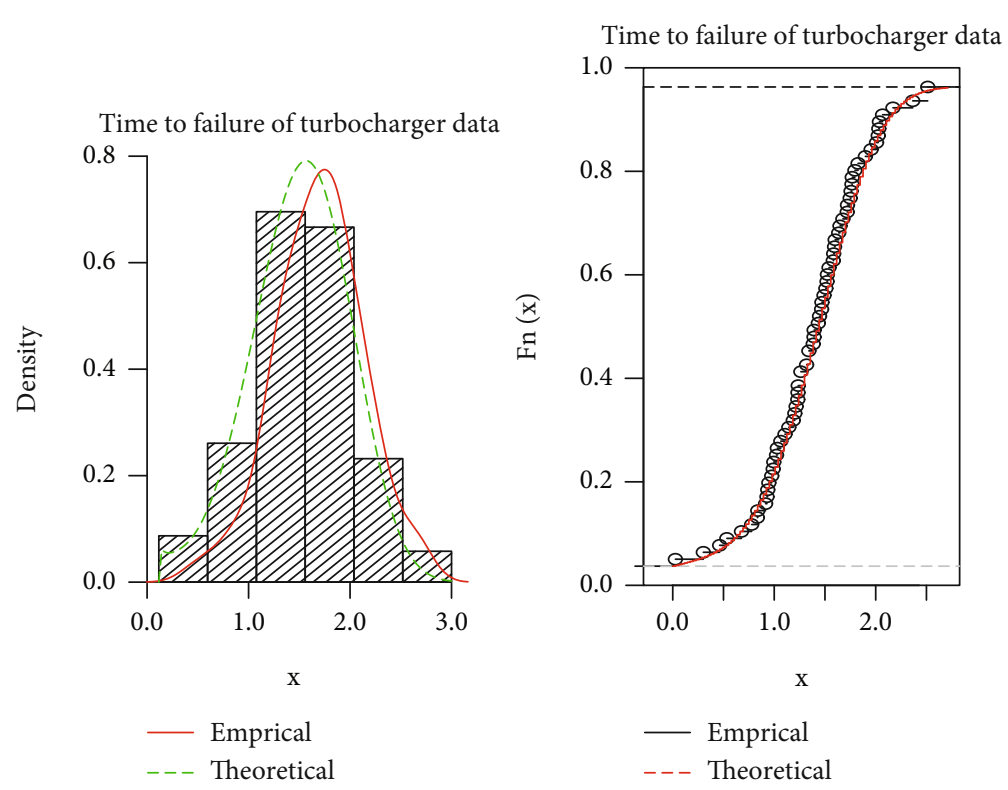

FIgURE 4: Plots of the fitted empirical pdf and empirical cdf versus estimated pdf and estimated cdf for data set 1. 

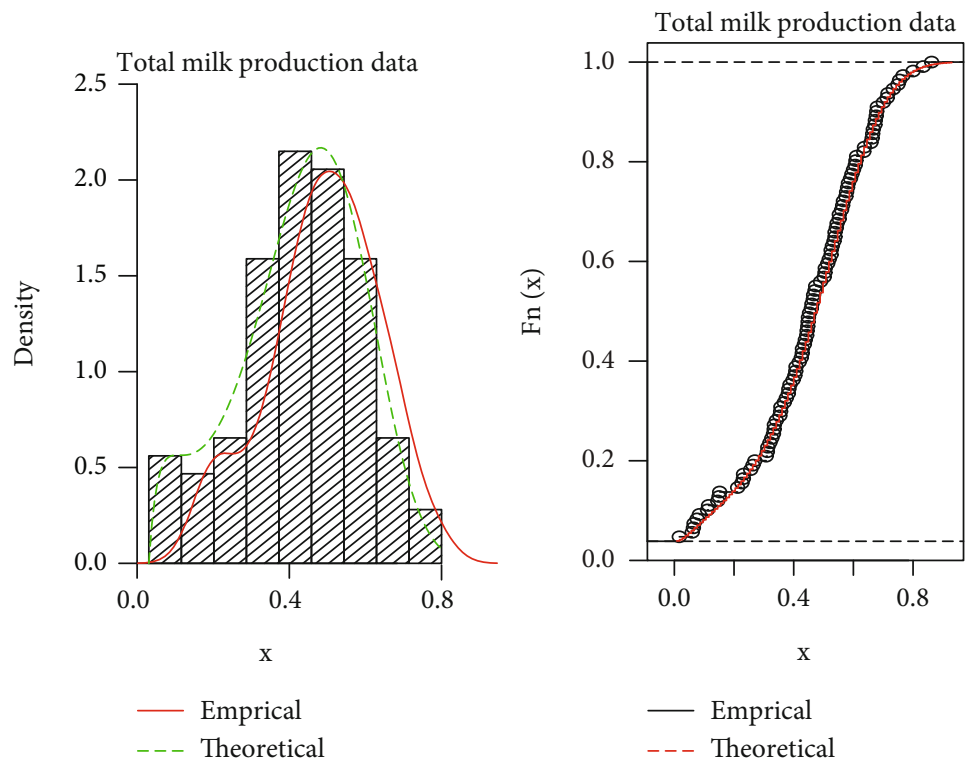

Figure 5: Plots of the fitted empirical pdf and empirical cdf versus estimated pdf and estimated cdf for data set 2.
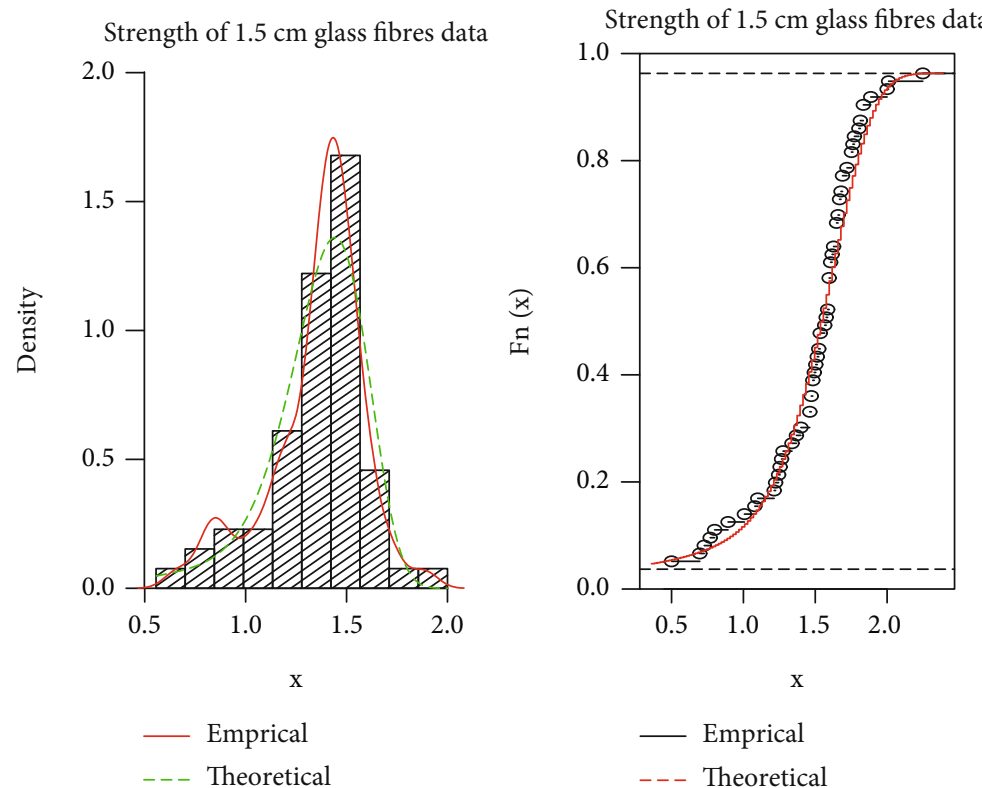

Figure 6: Plots of the fitted empirical pdf and empirical cdf versus estimated pdf and estimated cdf for data set 3.

$\left.U_{1}, U_{3}\right) \leq U_{3}$ and $\min \left(U_{2}, U_{3}\right) \leq U_{3}$, implying that $T \leq$ $U_{3}$ and so $F_{U_{3}}(x) \leq F_{T}(x)$. For the right inequality, based on the definition of $T$ in (2), it is clear that $T \geq \min \left(U_{1}\right.$ , $\left.U_{3}\right)$ and $T \geq \min \left(U_{2}, U_{3}\right)$. Therefore, for $x>0$,

$$
\begin{aligned}
F_{T}(x) & \leq \min \left[P\left(\min \left(U_{1}, U_{3}\right) \leq x\right), P\left(\min \left(U_{2}, U_{3}\right) \leq x\right)\right] \\
& =\min \left(1-e^{-\lambda_{1} x} e^{-\beta x^{\theta}}, 1-e^{-\lambda_{2} x} e^{-\beta x^{\theta}}\right) \\
& =1-e^{-\lambda_{*} x} e^{-\beta x^{\theta}}=G(x) .
\end{aligned}
$$

Proposition 3 is proved.
3.2. Generation of Numbers. Two different approaches are possible to generate values from the SCS distribution.

(i) First approach: analytical point of view. One can generate $n$ values from the unit uniform distribution, say $x_{1}, \cdots, x_{n}$. Then, $n$ values from the SCS distribution, say $t_{1}, \cdots, t_{n}$, are obtained by solving numerically the following equation: $x_{i}=F_{T}\left(t_{i}\right)$

(ii) Second approach: computational point of view. Based on the definition of $T$ in (2), one can generate $n$ values from $U_{1}$, say $u_{1,1}, \cdots, u_{1, n}, n$ values from $U_{2}$, say $u_{2,1}, \cdots, u_{2, n}$ and $n$ values from $U_{3}$, say $u_{3,1}, \cdots$, $u_{3, n}$. Then, $n$ values from the SCS distribution, say 


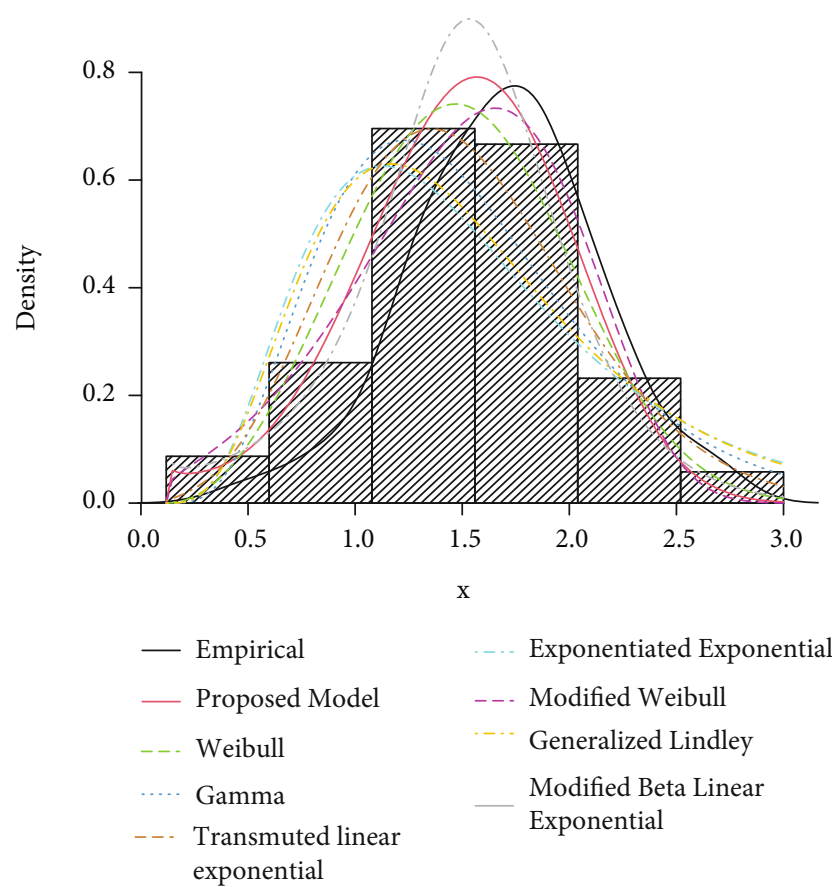

Figure 7: Plots of the fitted pdfs with the empirical pdf and the histograms of data set 1 .

$$
\begin{aligned}
& t_{1}, \cdots, t_{n} \text {, are obtained by taking: } t_{i}=\max \left[\operatorname { m i n } \left(u_{1, i}\right.\right. \\
& \left.\left., u_{3, i}\right), \min \left(u_{2, i}, u_{3, i}\right)\right]
\end{aligned}
$$

3.3. Moments. Diverse moments of the SCS distributions are now discussed. For this purpose, let us introduce the following special integral:

$$
\mathscr{I}_{\beta, \theta}(a, b, t)=\int_{0}^{t} x^{a} e^{-b x} e^{-\beta x^{\theta}} \mathrm{d} x
$$

Firstly, this integral is well defined for $a>-1, b>0$, and $t \geq 0$ (other combinations of parameters are possible but out of the scope of this study). Based on the series expansion of the exponential functions, the following expansions hold, depending on the possible values of $\theta$.

(i) When $\theta \geq 1$, we can expand $\mathscr{I}_{\beta, \theta}(a, b, t)$ as

$$
\mathscr{I}_{\beta, \theta}(a, b, t)=\frac{1}{\theta} \frac{1}{\beta^{(a+1) / \theta}} \sum_{k=0}^{+\infty} \frac{(-b)^{k}}{k !} \frac{1}{\beta^{k / \theta}} \gamma\left((k+a+1) / \theta, \beta t^{\theta}\right),
$$

where $\gamma(a, x)=\int_{0}^{x} y^{a-1} e^{-y} \mathrm{~d} y, a, x>0$.

(ii) When $\theta \in(0,1)$, we can expand $\mathscr{I}_{\beta, \theta}(a, b, t)$ as

$$
\mathscr{I}_{\beta, \theta}(a, b, t)=\frac{1}{b^{a+1}} \sum_{k=0}^{+\infty} \frac{(-\beta)^{k}}{k !} \frac{1}{b^{\theta k}} \gamma(a+\theta k+1, b t)
$$

We can apply $t \longrightarrow+\infty$; by the ratio test, one can show that these two series expansions converge. Every incomplete moment of $T$ can be expressed according to a finite combination of special integrals above, with specific parameters. This is formulated in the proposition below.

Proposition 4. For $t \geq 0$, the $r^{\text {th }}$ incomplete moment of $T$ at $t \geq 0$ is given as

$$
\begin{aligned}
m_{r}(t)= & E\left(T^{r} I(\{T \leq t\})\right)=\beta \theta \mathscr{I}_{\beta, \theta}\left(r+\theta-1, \lambda_{1}, t\right) \\
& +\lambda_{1} \mathscr{I}_{\beta, \theta}\left(r, \lambda_{1}, t\right)+\beta \theta \mathscr{I}_{\beta, \theta}\left(r+\theta-1, \lambda_{2}, t\right) \\
& +\lambda_{2} \mathscr{I}_{\beta, \theta}\left(r, \lambda_{2}, t\right)-\beta \theta \mathscr{I}_{\beta, \theta}\left(r+\theta-1, \lambda_{1}+\lambda_{2}, t\right) \\
& -\left(\lambda_{1}+\lambda_{2}\right) \mathscr{I}_{\beta, \theta}\left(r, \lambda_{1}+\lambda_{2}, t\right),
\end{aligned}
$$

where $I(A)$ denotes the indicator function over the event $A$.

Proof. The proof can be conducted by using the definition of $f_{T}(x)$ specified in (5). Indeed, we have

$$
\begin{aligned}
m_{r}(t)= & \int_{0}^{t} x^{r} f_{T}(x) \mathrm{d} x=\beta \theta \int_{0}^{t} x^{r+\theta-1} e^{-\lambda_{1} x} e^{-\beta x^{\theta}} \mathrm{d} x+\lambda_{1} \int_{0}^{t} x^{r} e^{-\lambda_{1} x} e^{-\beta x^{\theta}} \mathrm{d} x+\beta \theta \\
& \int_{0}^{t} x^{r+\theta-1} e^{-\lambda_{2} x} e^{-\beta x^{\theta}} \mathrm{d} x+\lambda_{2} \int_{0}^{t} x^{r} e^{-\lambda_{2} x} e^{-\beta x^{\theta}} \mathrm{d} x-\beta \theta \\
& \int_{0}^{t} x^{r+\theta-1} e^{-\left(\lambda_{1}+\lambda_{2}\right) x} e^{-\beta x^{\theta}} \mathrm{d} x-\left(\lambda_{1}+\lambda_{2}\right) \int_{0}^{t} x^{r} e^{-\left(\lambda_{1}+\lambda_{2}\right) x} e^{-\beta x^{\theta}} \mathrm{d} x \\
= & \beta \theta \mathscr{I}_{\beta, \theta}\left(r+\theta-1, \lambda_{1}, t\right)+\lambda_{1} \mathscr{I}_{\beta, \theta}\left(r, \lambda_{1}, t\right)+\beta \theta \mathscr{I}_{\beta, \theta}\left(r+\theta-1, \lambda_{2}, t\right) \\
& \quad+\lambda_{2} \mathscr{I}_{\beta, \theta}\left(r, \lambda_{2}, t\right)-\beta \theta \mathscr{I}_{\beta, \theta}\left(r+\theta-1, \lambda_{1}+\lambda_{2}, t\right) \\
& \quad-\left(\lambda_{1}+\lambda_{2}\right) \mathscr{I}_{\beta, \theta}\left(r, \lambda_{1}+\lambda_{2}, t\right) .
\end{aligned}
$$

This ends the proof of Proposition 4 .

From the incomplete moments of $T$, one can derive several measures of $T$ and functions of interest. For instance, the $r^{\text {th }}$ moment of $T$ also follows from the $r^{\text {th }}$ incomplete moment of $X$ by applying $t \longrightarrow+\infty$. Owing to (19), we can write

$$
\begin{aligned}
m_{r}=E\left(T^{r}\right)= & \lim _{t} m_{r}(t)=\beta \theta \mathscr{I}_{\beta, \theta}\left(r+\theta-1, \lambda_{1},+\infty\right) \\
& +\lambda_{1} \mathscr{I}_{\beta, \theta}\left(r, \lambda_{1},+\infty\right)+\beta \theta \mathscr{I}_{\beta, \theta}\left(r+\theta-1, \lambda_{2},+\infty\right) \\
& +\lambda_{2} \mathscr{I}_{\beta, \theta}\left(r, \lambda_{2},+\infty\right)-\beta \theta \mathscr{I}_{\beta, \theta}\left(r+\theta-1, \lambda_{1}+\lambda_{2},+\infty\right) \\
& -\left(\lambda_{1}+\lambda_{2}\right) \mathscr{I}_{\beta, \theta}\left(r, \lambda_{1}+\lambda_{2},+\infty\right) .
\end{aligned}
$$

Note that, since $T \leq U_{3}$, the following inequality holds: $m_{r} \leq \beta^{-r / \theta} \Gamma(r / \theta+1)$, where $\Gamma(a)=\lim _{x \longrightarrow+\infty} \gamma(a, x)$.

The mean and standard deviation of $T$ are obtained as $\mu=m_{1}$ and $\sigma=\left(m_{2}-m_{1}^{2}\right)^{1 / 2}$. The skewness coefficient of $T$ 


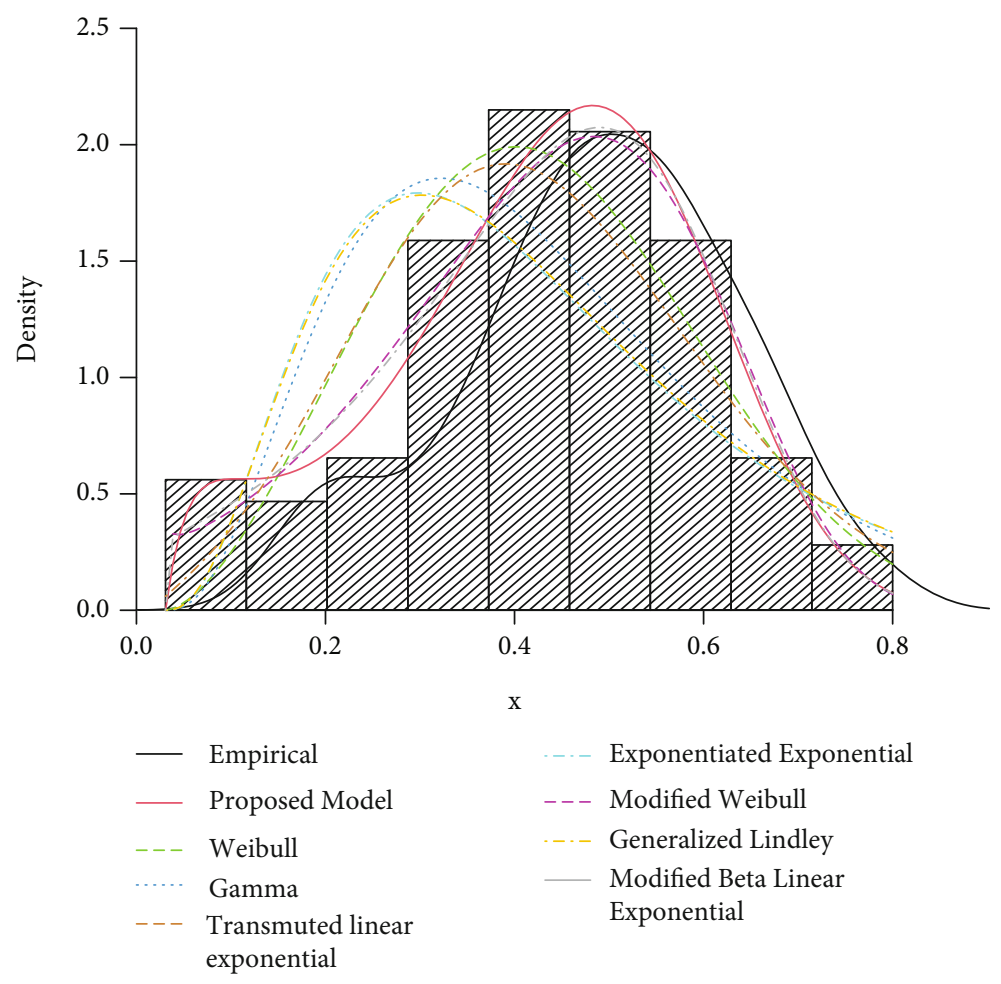

Figure 8: Plots of the fitted pdfs with the empirical pdf and the histograms of data set 2.

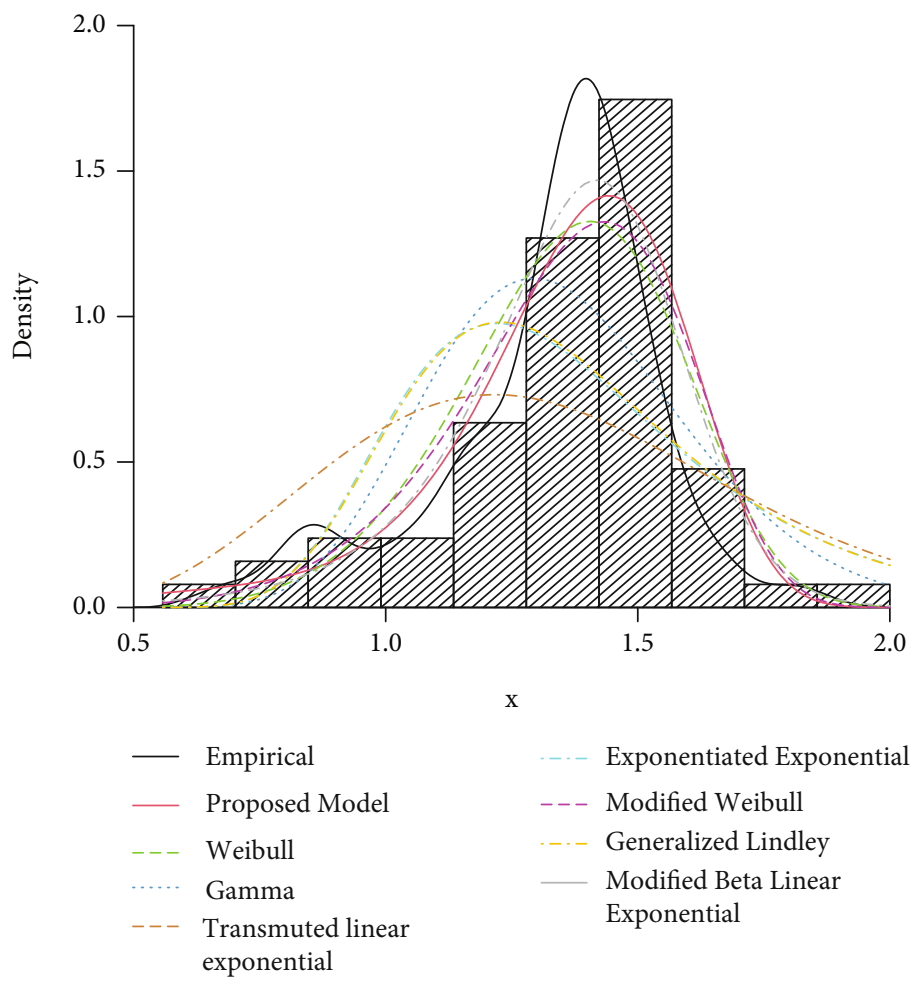

Figure 9: Plots of the fitted pdfs with the empirical pdf and the histograms of data set 3. 


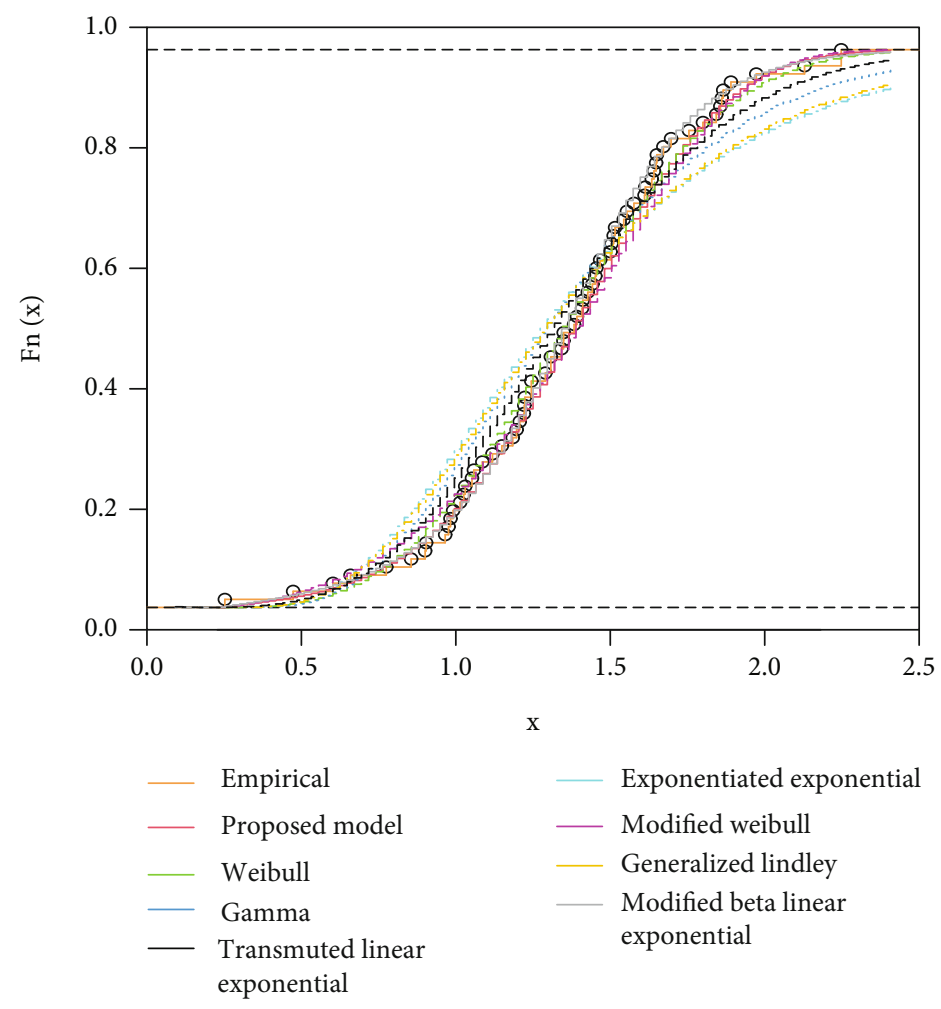

Figure 10: Plots of the fitted cdfs versus empirical cdf of data set 1.

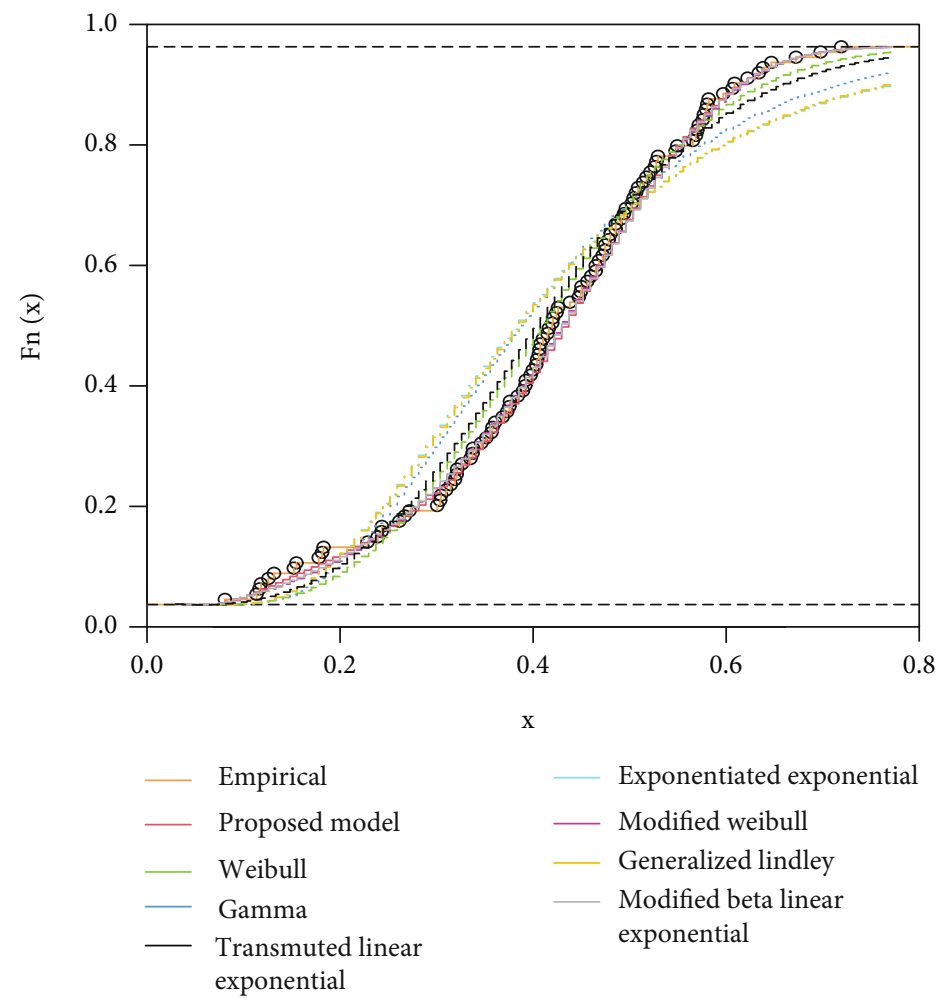

Figure 11: Plots of the fitted cdfs versus empirical cdf of data set 2. 


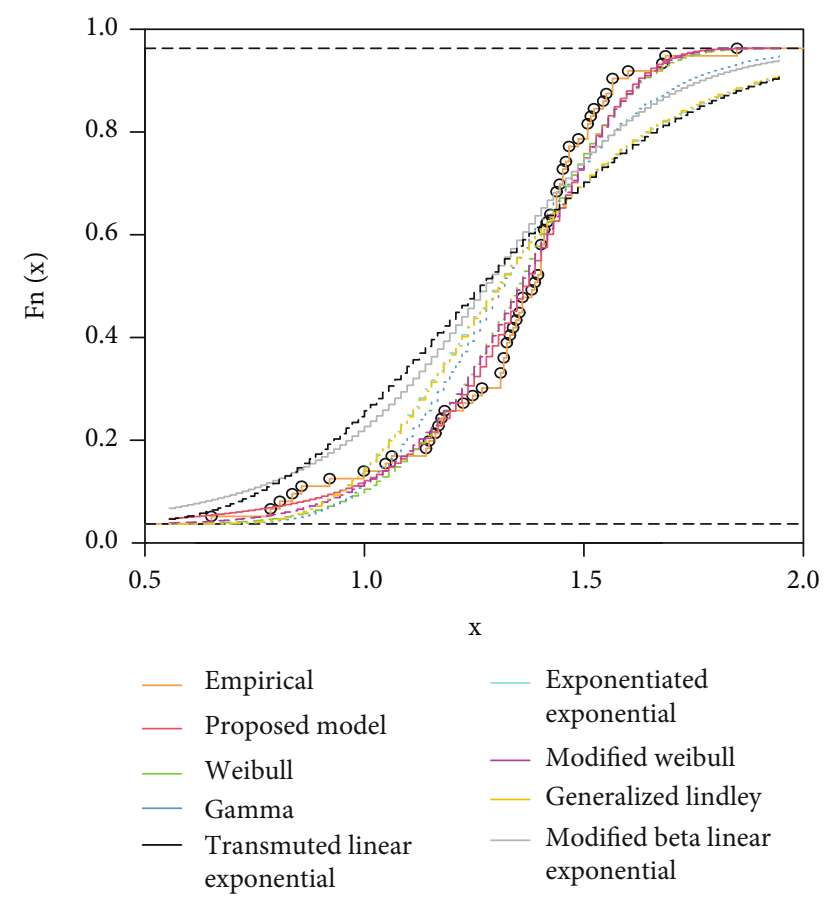

Figure 12: Plots of the fitted cdfs versus empirical cdf of data set 3.

can be determined as

$$
S=\sigma^{-3} E\left((T-\mu)^{3}\right)=6 \sigma^{-3} \sum_{k=0}^{3} \frac{1}{k !(3-k) !}(-1)^{3-k} \mu^{3-k} m_{k}
$$

and the kurtosis coefficient of $T$ is given as

$$
K=\sigma^{-4} E\left((T-\mu)^{4}\right)=24 \sigma^{-4} \sum_{k=0}^{4} \frac{1}{k !(4-k) !}(-1)^{4-k} \mu^{4-k} m_{k} .
$$

Also, from (19), one can define conditional moments, residual life, mean deviations, and several reliability curves (see, for instance, [9]). For instance, the $r^{\text {th }}$ conditional moment of $T$ at $t \geq 0$ is obtained as

$$
m_{r}^{*}(t)=E\left(T^{r} \mid T>t\right)=\frac{1}{S_{T}(t)}\left[m_{r}-m_{r}(t)\right]
$$

which is fully expressible in terms of combinations of special integrals.

3.4. Moment Generating Function. The moment generating function of $T$ can be expressed in terms of linear combinations of special integral functions, as developed below.
Proposition 5. For $s \leq \min \left(\lambda_{1}, \lambda_{2}\right)$, the moment generating function of $T$ is given as

$$
\begin{aligned}
M(s)= & E\left(e^{s T}\right)=\beta \theta \mathscr{I}_{\beta, \theta}\left(\theta-1, \lambda_{1}-s,+\infty\right)+\lambda_{1} \mathscr{I}_{\beta, \theta}\left(0, \lambda_{1}-s,+\infty\right) \\
& +\beta \theta \mathscr{I}_{\beta, \theta}\left(\theta-1, \lambda_{2}-s,+\infty\right)+\lambda_{2} \mathscr{I}_{\beta, \theta}\left(0, \lambda_{2}-s,+\infty\right) \\
& -\beta \theta \mathscr{I}_{\beta, \theta}\left(\theta-1, \lambda_{1}+\lambda_{2}-s,+\infty\right)-\left(\lambda_{1}+\lambda_{2}\right) \mathscr{I}_{\beta, \theta}\left(0, \lambda_{1}+\lambda_{2}-s,+\infty\right)
\end{aligned}
$$

Proof. The proof exploits the definition of $f_{T}(x)$ determined in (5). We have

$$
\begin{aligned}
M(s)= & \int_{0}^{+\infty} e^{s x} f_{T}(x) \mathrm{d} x=\beta \theta \int_{0}^{+\infty} x^{\theta-1} e^{-\left(\lambda_{1}-s\right) x} e^{-\beta x^{\theta}} \mathrm{d} x+\lambda_{1} \\
& \int_{0}^{+\infty} e^{-\left(\lambda_{1}-s\right) x} e^{-\beta x^{\theta}} \mathrm{d} x+\beta \theta \int_{0}^{+\infty} x^{\theta-1} e^{-\left(\lambda_{2}-s\right) x} e^{-\beta x^{\theta}} \mathrm{d} x+\lambda_{2} \\
& \int_{0}^{+\infty} e^{-\left(\lambda_{2}-s\right) x} e^{-\beta x^{\theta}} \mathrm{d} x-\beta \theta \int_{0}^{+\infty} x^{\theta-1} e^{-\left(\lambda_{1}+\lambda_{2}-s\right) x} e^{-\beta x^{\theta}} \mathrm{d} x-\left(\lambda_{1}+\lambda_{2}\right) \\
& \int_{0}^{+\infty} e^{-\left(\lambda_{1}+\lambda_{2}-s\right) x} e^{-\beta x^{\theta}} \mathrm{d} x \\
= & \beta \theta \mathscr{I}_{\beta, \theta}\left(\theta-1, \lambda_{1}-s,+\infty\right)+\lambda_{1} \mathscr{I}_{\beta, \theta}\left(0, \lambda_{1}-s,+\infty\right) \\
& \quad+\beta \theta \mathscr{I}_{\beta, \theta}\left(\theta-1, \lambda_{2}-s,+\infty\right)+\lambda_{2} \mathscr{I}_{\beta, \theta}\left(0, \lambda_{2}-s,+\infty\right) \\
& \quad-\beta \theta \mathscr{I}_{\beta, \theta}\left(\theta-1, \lambda_{1}+\lambda_{2}-s,+\infty\right) \\
& \quad-\left(\lambda_{1}+\lambda_{2}\right) \mathscr{I}_{\beta, \theta}\left(0, \lambda_{1}+\lambda_{2}-s,+\infty\right) .
\end{aligned}
$$

This ends the proof of Proposition 5.

The characteristic function of $T$ is given as $\varphi_{T}(s)=E($ $\left.e^{\text {is } T}\right)=M($ is $)$, where $i^{2}=-1$, with no particular restriction on $s$. The moment generating and characteristic functions can be used for distributional results on the SCS distribution.

\section{Inference for the SCS Model with a Simulation Study}

The SCS model is defined by the cdf and pdf given as (3) and (5), respectively, under the assumption that the parameters $\lambda_{1}, \lambda_{2}, \beta$, and $\theta$ are unknown. Based on data, we aim to estimate these parameters. In this regard, we employ the famous maximum likelihood method (see, for instance, [10]). In the context of the SCS model, the essential is described below. Let $t_{1}, \cdots, t_{n}$ be $n$ observations of $T$ as defined by (2). Then, the maximum likelihood estimators (MLEs) of $\lambda_{1}, \lambda_{2}, \beta$, and $\theta$, denoted by $\widehat{\lambda}_{1}, \widehat{\lambda}_{2}, \widehat{\beta}$, and $\widehat{\theta}$, are determined through the following maximization procedure:

$$
\left(\widehat{\lambda}_{1}, \widehat{\lambda}_{2}, \widehat{\beta}, \widehat{\theta}\right)=\operatorname{argmax}_{\left(\lambda_{1}, \lambda_{2}, \beta, \theta\right) \in(0,+\infty)^{4}} \ell\left(\lambda_{1}, \lambda_{2}, \beta, \theta\right),
$$

where $\ell\left(\lambda_{1}, \lambda_{2}, \beta, \theta\right)$ refers to the log-likelihood function, which can be expressed as 


$$
\begin{aligned}
\ell\left(\lambda_{1}, \lambda_{2}, \beta, \theta\right)= & \sum_{i=1}^{n} \log \left[f_{T}\left(t_{i}\right)\right] \\
= & -\beta \sum_{i=1}^{n} t_{i}^{\theta}+\sum_{i=1}^{n} \log \left[\left(\beta \theta t_{i}^{\theta-1}+\lambda_{1}\right) e^{-\lambda_{1} t_{i}}\right. \\
& +\left(\beta \theta t_{i}^{\theta-1}+\lambda_{2}\right) e^{-\lambda_{2} t_{i}} \\
& \left.-\left(\beta \theta t_{i}^{\theta-1}+\lambda_{1}+\lambda_{2}\right) e^{-\left(\lambda_{1}+\lambda_{2}\right) t_{i}}\right]
\end{aligned}
$$

As usual, the MLEs can be determined numerically through the use of any mathematical software. Here, the $R$ software is used (see [11]). Now, we perform simulation studies to verify whether the maximum likelihood method is appropriate for estimating the parameters of the proposed model and also to illustrate the performance of the associated estimates. In this regard, random number generation is carried out using the first approach described in Subsection 3.2. We consider sample sizes $n=25,50,100,300$, and 500 for the following four random parameter combinations:

$\left(\lambda_{1}=0.1, \lambda_{2}=0.2, \beta=0.1, \theta=0.3\right),\left(\lambda_{1}=0.5, \lambda_{2}=0.8, \beta=0.6, \theta=0.9\right)$, $\left(\lambda_{1}=1.2, \lambda_{2}=1.2, \beta=0.9, \theta=0.6\right),\left(\lambda_{1}=1.6, \lambda_{2}=1.8, \beta=1.4, \theta=1.5\right)$.

This procedure is repeated $N=1000$ times for calculation of bias, variance (Var), mean squared error (MSE), and coverage probability $(\mathrm{CP})$. The generic formulas to estimate bias, variance, and MSE are given by

$$
\begin{aligned}
& \operatorname{Bias}\left(\Theta_{i}\right)=\frac{1}{N} \sum_{J=1}^{N}\left(\widehat{\Theta}_{i, j}-\Theta_{i}\right), \quad \operatorname{MSE}\left(\Theta_{i}\right)=\frac{1}{N} \sum_{J=1}^{N}\left(\Theta \wedge_{i, j}-\Theta_{i}\right)^{2}, \\
& \operatorname{Var}\left(\Theta_{i}\right)=\frac{1}{N} \sum_{J=1}^{N}\left(\Theta \wedge_{i, j}-\bar{\Theta}_{i}\right)^{2},
\end{aligned}
$$

where $\Theta_{1}=\lambda_{1}, \Theta_{2}=\lambda_{2}, \Theta_{3}=\beta, \Theta_{4}=\theta, i=1,2,3,4$, and $\widehat{\Theta}_{i, j}$ are the MLE of $\Theta_{i}$ at the $j^{\text {th }}$ repetition, and $\bar{\Theta}_{i}$ is the average MLEs value for the $N$ repetitions,

$C P\left(\Theta_{i}\right)=\frac{1}{N} \sum_{j=1}^{N} I\left(\widehat{\Theta}_{i, j}-Z_{\alpha / 2} S E\left(\widehat{\Theta}_{i, j}\right), \widehat{\Theta}_{i, j}+Z_{\alpha / 2} S E\left(\widehat{\Theta}_{i, j}\right)\right)$,

where $I(a, b)$ is the indicator function, defined as $I(a, b)=1$ if $\Theta_{i} \in[a, b]$, and 0; otherwise, $Z_{\alpha / 2}$ is the two tailed critical value of standard normal distribution at $\alpha(0.05)$ level of significance, i.e., $Z_{\alpha / 2}=1.9599$ and $\operatorname{SE}\left(\widehat{\Theta}_{i, j}\right)$ is the estimate of the standard error related to $\widehat{\Theta}_{i, j}$.

The numerical results of the simulation are given in Tables 1 and 2.

It is clear from Tables 1 and 2 that the estimated biases, variances, and MSEs decrease when the sample size $n$ increases. Thus, the simulation study shows that the maximum likelihood method is appropriate for estimating the parameters of the proposed distribution. The MSEs of the parameters tend to be closer to zero when $n$ increases. Also, this reveals the consistency property of the MLEs. Hence, it is expected that the MLEs are going to work fine when the model is applied to real life situations, as shown in the next section. Moreover, the coverage probabilities (CPs) are near to 0.95 and approach to the nominal value when the sample size increases.

\section{Application}

In this section, we explore the application of the newly introduced SCS model in comparison to Weibull, gamma, exponentiated exponential, modified Weibull (see [12]), generalized Lindley (see [13]), transmuted linear exponential (see [14]), and modified beta linear exponential models (see [15]).

We first analyze the data set representing the time to failure of turbocharger (see [16]) of a certain type of engine. The data set, called data set 1 , is given as follows: 0.0312 , $0.314,0.479,0.552,0.700,0.803,0.861,0.865,0.944,0.958$, $0.966,0.977,1.006,1.021,1.027,1.055,1.063,1.098,1.140$, $1.179,1.224,1.240,1.253,1.270,1.272,1.274,1.301,1.301$, $1.359,1.382,1.382,1.426,1.434,1.435,1.478,1.490,1.511$, $1.514,1.535,1.554,1.566,1.570,1.586,1.629,1.633,1.642$, $1.648,1.684,1.697,1.726,1.770,1.773,1.800,1.809,1.818$, $1.821,1.848,1.880,1.954,2.012,2.067,2.084,2.090,2.096$, $2.128,2.233,2.433,2.585$, and 2.585 .

Descriptive statistics of the data set 1 are given in Table 3 and are calculated using moments package in $R$ software (version 3.5.3).

The average time to failure of a turbocharger is 1.447 with a standard deviation of 0.506 . The skewness and kurtosis coefficients for the given data are -0.164 and 3.236, respectively, implying that data set 1 has slight negative skewness and is leptokurtic. It is also clear that the data are under dispersed, with an index of dispersion of 0.177 .

The second data set to be analyzed represents the total milk production in the first birth of 107 cows from the SINDI race. These cows are property of the Carnaúba farm which belongs to the Agropecuária Manoel Dantas Ltda (AMDA), located in Taperoá City, Paraba (Brazil). This data is presented by [17]. The data set, called data set 2 , is given as follows: $0.4365,0.4260,0.5140,0.6907,0.7471,0.2605$, $0.6196,0.8781,0.4990,0.6058,0.6891,0.5770,0.5394$, $0.1479,0.2356,0.6012,0.1525,0.5483,0.6927,0.7261$, $0.3323,0.0671,0.2361,0.4800,0.5707,0.7131,0.5853$, $0.6768,0.5350,0.4151,0.6789,0.4576,0.3259,0.2303$, $0.7687,0.4371,0.3383,0.6114,0.3480,0.4564,0.7804$, $0.3406,0.4823,0.5912,0.5744,0.5481,0.1131,0.7290$, $0.0168,0.5529,0.4530,0.3891,0.4752,0.3134,0.3175$, $0.1167,0.6750,0.5113,0.5447,0.4143,0.5627,0.5150$, $0.0776,0.3945,0.4553,0.4470,0.5285,0.5232,0.6465$, $0.0650,0.8492,0.8147,0.3627,0.3906,0.4438,0.4612$, $0.3188,0.2160,0.6707,0.6220,0.5629,0.4675,0.6844$, $0.3413,0.4332,0.0854,0.3821,0.4694,0.3635,0.4111$, $0.5349,0.3751,0.1546,0.4517,0.2681,0.4049,0.5553$, $0.5878,0.4741,0.3598,0.7629,0.5941,0.6174,0.6860$, $0.0609,0.6488$, and 0.2747 . 
Descriptive statistics of the data set 2 are given in Table 4.

The average milk production in the first birth of 107 cows from the SINDI race is 0.469 , with a standard deviation of 0.192 . The skewness and kurtosis coefficients for the given data are -0.335 and 2.686, respectively, implying that the data set 2 has a slightly negative skewness with platykurtic nature. It is also clear that the data are under dispersed, with an index of dispersion of 0.079 .

The third data set to be analyzed represents the strength of $1.5 \mathrm{~cm}$ glass fibers, measured at the National physical laboratory, England reported in [18]. The data was originally used by [19]. The data are $0.55,0.93,1.25,1.36,1.49,1.52$, $1.58,1.61,1.64,1.68,1.73,1.81,2.00,0.74,1.04,1.27,1.39$, $1.49,1.53,1.59,1.61,1.66,1.68,1.76,1.82,2.01,0.77,1.11$, $1.28,1.42,1.50,1.54,1.60,1.62,1.66,1.69,1.76,1.84,2.24$, $0.81,1.13,1.29,1.48,1.50,1.55,1.61,1.62,1.66,1.70,1.77$, $1.84,0.84,1.24,1.30,1.48,1.51,1.55,1.61,1.63,1.67,1.70$, 1.78 , and 1.89 . The summary statistics of the data set 3 is given in Table 5 .

The average strength of $1.5 \mathrm{~cm}$ glass fibers is 1.507 , with a standard deviation of 0.324 . The skewness and kurtosis coefficients for the given data are -0.899 and 3.923 , respectively, thus implying that the data set 3 has a slight negative skewness with leptokurtic nature. Furthermore, we see that the data are under dispersed, with an index of dispersion of 0.069 .

The MLEs are computed using the Nelder-Mead optimization method (see [20]), and the log-likelihood function is evaluated. Nelder-Mead optimization for getting MLEs was executed through $R$ software version 3.5.3 using the MASS package. The MLEs of the parameters along with standard errors of parameters in parenthesis are reported in Tables 6-8 for data sets 1,2 , and 3, respectively.

The goodness-of-fit measures, the value for the Kolmogorov-Smirnov $(K-S)$ statistic, Anderson-Darling (AD), Cramér-von Mises (CVM), and their $p$ values are reported.

Tables 9-11 exhibit the $K-S$ with its $p$ values, AD statistic with its $p$ values, and CVM statistic with its $p$ values for the models fitted to the data sets 1,2, and 3, respectively.

On the basis of inferential statistical tests, the $p$ values associated to $K-S, \mathrm{AD}$, and CVM are statistically supporting the null hypothesis mainly for the proposed model as compared to other competing models for all the fitted real-life data sets (see Tables 9-11). We note that some comparative models in Table 11 may not be fitted to the data set 3 (based on their $p$ values) but we use them only for comparison purposes.

For graphical observation of the fitted SCS model to reallife data sets, the plots of the fitted empirical pdf and empirical cdf versus estimated pdf (plotted based on the density of the distribution with replacing its parameters by their MLEs) and estimated cdf for data sets 1,2 , and 3 are given in Figures 4-6, respectively. Just like we create a histogram from the data points, in a similar fashion, "empirical pdf" is generated from the data points of the data set. For comparison purposes, just like we check, which model curve is close to the histogram, in a similar way, we check which model curve is close to the "empirical pdf" curve.
We observe very little deviance between empirical and estimated curves in the three data sets.

Plots of the fitted pdfs of all the models with the empirical pdfs and the histograms of data sets 1,2, and 3 are given in Figures 7-9, respectively.

From Figures 7-9, we note that the proposed model pdf is closer to fit the data sets as compared to other competing models.

Plot of fitted cdfs of all the models versus empirical cdf for data sets 1, 2, and 3 is given in Figures 10-12, respectively.

Based on Figures 10-12, we see that the fitted cdf curve for the proposed model has least deviance with respect to empirical cdf of the data sets as compared to other competing models.

\section{Conclusion}

In this paper, based on the notion of a coherent system, a new four-parameter probability distribution was introduced, called the SCS distribution. Several of its structural properties are studied based on its max-min definition, including the analysis of its main functions, diverse stochastic orders, moments, and moment generating functions. We discuss the fact that the SCS distribution is sufficiently pliant for diverse statistical purposes, including data fitting. In particular, it shows a significant role for fitting data sets having slight negative skewness with platykurtic as well as leptokurtic nature. With the consideration of three real-life data sets, two in reliability and another in production, this aspect is emphasized, showing that the SCS model outperforms several comparable models. Hence, we believe that the SCS model has the qualities to join the arsenal of lifetime models used for deep analysis of data.

\section{Data Availability}

All the study data is available on request.

\section{Conflicts of Interest}

The authors declare that there are no conflicts of interest regarding the publication of this paper.

\section{Acknowledgments}

The authors appreciate the detailed remarks of the referees, which helped to improve the quality of the article.

\section{References}

[1] A. Hoyland and M. Rausand, System reliability theory: models and statistical methods, John Wiley and Sons, 2009.

[2] P. J. Boland and F. J. Samaniego, "The signature of a coherent system and its applications in reliability," Mathematical Reliability: An Expository Perspective, vol. 67, pp. 3-30, 2004.

[3] R. E. Barlow, F. Proschan, and L. C. Hunter, Mathematical theory of reliability, Cambridge University Press, 1996.

[4] G. E. Baggs and H. N. Nagaraja, "Reliability properties of order statistics from bivariate exponential distributions," 
Communications in Statistics. Stochastic Models, vol. 12, no. 4, pp. 611-631, 1996.

[5] H. W. Block, Y. Li, and T. H. Savits, "Initial and final behaviour of failure rate functions for mixtures and systems," Journal of Applied Probability, vol. 40, no. 3, pp. 721-740, 2003.

[6] R. C. Gupta, "Reliability studies of bivariate distributions with Pareto conditionals," Journal of Multivariate Analysis, vol. 76, no. 2, pp. 214-225, 2001.

[7] P. L. Gupta and R. C. Gupta, "Failure rate of the minimum and maximum of a multivariate normal distribution," Metrika, vol. 53, no. 1, pp. 39-49, 2001.

[8] S. Eryilmaz, "Multivariate stress-strength reliability model and its evaluation for coherent structures," Journal of Multivariate Analysis, vol. 99, no. 9, pp. 1878-1887, 2008.

[9] G. M. Cordeiro, R. B. Silva, and A. D. C. Nascimento, Recent advances in lifetime and reliability models, Bentham books, 2020.

[10] G. Casella and R. L. Berger, Statistical Inference, Brooks/Cole Publishing Company, California, 1990.

[11] R Core Team, "R version 3.5.3: a language and environment for statistical computing," R Foundation for Statistical Computing, Vienna, Austria, 2019, https://www.R-project.org/.

[12] C. Lai, M. Xie, and D. N. P. Murthy, "A modified Weibull distribution,” IEEE Transactions on Reliability, vol. 52, no. 1, pp. 33-37, 2003.

[13] S. Nadarajah, H. S. Bakouch, and R. Tahmasbi, "A generalized Lindley distribution," Sankhya B, vol. 73, no. 2, pp. 331-359, 2011.

[14] Y. Tian, M. Tian, and Q. Zhu, "Transmuted linear exponential distribution: a new generalization of the linear exponential distribution," Communications in Statistics - Simulation and Computation, vol. 43, no. 10, pp. 2661-2677, 2014.

[15] H. S. Bakouch, A. Saboor, and M. N. Khan, "Modified beta linear exponential distribution with hydrologic applications," Annals of Data Science, vol. 8, no. 1, pp. 131-157, 2019.

[16] I. Elbatal, F. Jamal, C. Chesneau, M. Elgarhy, and S. Alrajhi, "The modified beta Gompertz distribution: theory and applications," Mathematics, vol. 7, no. 1, p. 3, 2019.

[17] G. M. Cordeiro and R. D. S. Brito, "The beta power distribution," Brazilian Journal of Probability and Statistics, vol. 26, no. 1, pp. 88-112, 2012.

[18] N. Ekhosuehi and F. Opone, "A three parameter generalized Lindley distribution: properties and application," Statistica, vol. 78, no. 3, pp. 233-249, 2018.

[19] R. L. Smith and J. C. Naylor, “A comparison of maximum likelihood and Bayesian estimators for the three- parameter Weibull distribution," Journal of the Royal Statistical Society. Series C (Applied Statistics), vol. 36, no. 3, pp. 358-369, 1987.

[20] J. A. Nelder and R. Mead, "A simplex method for function minimization,” Computer Journal, vol. 7, no. 4, pp. 308-313, 1965. 